\title{
A novel risk-predicted nomogram for sepsis associated-acute kidney injury among critically ill patients
}

\author{
Shanglin Yang ${ }^{1 \dagger}$, Tingting Su ${ }^{2 \dagger}$, Lina Huang ${ }^{3}$, Lu-Huai Feng ${ }^{3^{*}}$ and Tianbao Liao ${ }^{4^{*}}$
}

\begin{abstract}
Background: Acute kidney injury (AKI) is a prevalent and severe complication of sepsis contributing to high morbidity and mortality among critically ill patients. In this retrospective study, we develop a novel risk-predicted nomogram of sepsis associated-AKI (SA-AKI).

Methods: A total of 2,871 patients from the Medical Information Mart for Intensive Care III (MIMIC-III) critical care database were randomly assigned to primary (2,012 patients) and validation (859 patients) cohorts. A risk-predicted nomogram for SA-AKI was developed through multivariate logistic regression analysis in the primary cohort while the nomogram was evaluated in the validation cohort. Nomogram discrimination and calibration were assessed using C-index and calibration curves in the primary and external validation cohorts. The clinical utility of the final nomogram was evaluated using decision curve analysis.

Results: Risk predictors included in the prediction nomogram included length of stay in intensive care unit (LOS in ICU), baseline serum creatinine ( $\mathrm{SCr}$ ), glucose, anemia, and vasoactive drugs. Nomogram revealed moderate discrimination and calibration in estimating the risk of SA-AKI, with an unadjusted C-index of $0.752,95 \% \mathrm{Cl}(0.730-$ 0.774), and a bootstrap-corrected $\mathrm{C}$ index of 0.749 . Application of the nomogram in the validation cohort provided moderate discrimination (C-index, 0.757 [95\% Cl, 0.724-0.790]) and good calibration. Besides, the decision curve analysis (DCA) confirmed the clinical usefulness of the nomogram.
\end{abstract}

Conclusions: This study developed and validated an AKI risk prediction nomogram applied to critically ill patients with sepsis, which may help identify reasonable risk judgments and treatment strategies to a certain extent. Nevertheless, further verification using external data is essential to enhance its applicability in clinical practice.

Keywords: Sepsis, Acute kidney injury, Prediction, Nomogram, Intensive care unit

\footnotetext{
*Correspondence: 823900187@qq.com; yyliaotianbao@126.com

†Shanglin Yang and Tingting Su contributed equally to this work.

${ }^{3}$ Department of Comprehensive Internal Medicine, The Affiliated Tumor

Hospital of Guangxi Medical University, Nanning, China

${ }^{4}$ Department of President's Office, YouJiang Medical University for Nationalities, Baise, China

Full list of author information is available at the end of the article
}

(C) The Author(s). 2021 Open Access This article is licensed under a Creative Commons Attribution 4.0 International License, which permits use, sharing, adaptation, distribution and reproduction in any medium or format, as long as you give appropriate credit to the original author(s) and the source, provide a link to the Creative Commons licence, and indicate if changes were made. The images or other third party material in this article are included in the article's Creative Commons licence, unless indicated otherwise in a credit line to the material. If material is not included in the article's Creative Commons licence and your intended use is not permitted by statutory regulation or exceeds the permitted use, you will need to obtain permission directly from the copyright holder. To view a copy of this licence, visit http://creativecommons.org/licenses/by/4.0/. The Creative Commons Public Domain Dedication waiver (http://creativecommons.org/publicdomain/zero/1.0/) applies to the data made available in this article, unless otherwise stated in a credit line to the data. 


\section{Background}

Sepsis is a major global cause of high morbidity and mortality for critically ill patients [1] with a continuously increasing incidence [2]. Besides, sepsis is an enormous burden, accounting for $\sim 850,000$ emergency visits per year and up to 381,000 annual related deaths in the USA $[2,3]$. Sepsis can lead to various complications. For instance, SA-AKI is a common and severe complication of sepsis that meets consensus criteria for both sepsis and AKI [4], indicating multiple organ dysfunction and significant poor clinical outcomes $[5,6]$. Noteworthy, sepsis is associated with up to $50 \%$ of AKI [7], and up to $60 \%$ of patients with sepsis have AKI [8]. Among critically ill patients with SA-AKI, the mortality rates range from 38.2 to $70.2 \%$ [8, 9]. Nevertheless, so far, no single effective therapy has been reported to change the outcome of SA-AKI [10]. Notably, early diagnosis and treatment improve the long-term outcome of patients. Therefore, early identification of high-risk patients is important for AKI prevention [11].

SA-AKI is diagnosed based on specific, contextdependent, and imperfect definitions, besides, an increase in serum creatinine or a decline in urine output remains its key diagnostic criteria [12]. Accumulating knowledge has highlighted the clinical risk factors, pathobiology, response to treatment, and elements of renal recovery thereby improving the prevention, detection, and treatment of SA-AKI [4]. Nonetheless, the pathogenesis of SAAKI remains completely unclear and its risk factors emerge from various sources, making it a major clinical challenge in early detection $[13,14]$. Despite many novel biomarkers related to the early diagnosis and prognosis of SA-AKI being reported, few are applied in clinical practice [15]. Recent studies have used the patient and disease characteristics of AKI to identify patients with an increased risk, however, most of them have not integrated these with clinical prediction models [16-19]. Other studies have developed risk assessment models for AKI in patients based on patient and disease characteristics alone $[20,21]$, yet few have been developed for SA-AKI. As such, there is an urgent need for a more modern framework for rapid clinical diagnosis of SA-AKI.

A nomogram is a reliable tool that predicts and quantifies risk for a clinical event by creating a visualized graph of the predictive model based on relevant factors [22, 23]. Herein, we identified a combination of routinely available clinical variables for a highly precise prediction of SA-AKI in critically ill patients.

\section{Methods}

\section{Database}

Retrospectively, data were extracted from the MIMIC-III database (version 1.4), a large US-based, publicly available critical care database [24]. The MIMIC-III (v1.4) database included unidentified health-related data of 61, 532 ICU stays at the Beth Israel Deaconess Medical Center between June 2001 and October 2012. The establishment of MIMIC-III (v1.4) was approved by the institutional review boards of the Beth Israel Deaconess Medical Center (Boston, MA) and Massachusetts Institute of Technology (Cambridge, MA), thus, this study was granted a waiver of informed consent. One author (L-H F) completed the online training course of the National Institutes of Health (certification number $35,897,462)$ to access the MIMIC-III (v1.4).

\section{Participants}

Adult patients ( $\geq 18$ years old) diagnosed with sepsis identified from the International Classification of Diseases 9th Edition (ICD-9) code were selected from the MIMIC-III v1.4 database. Patients with AKI before admission to the ICU were excluded. For patients with more than one ICU stay, only the first ICU admission of each patient was analyzed.

A pre-seeded random number (123) generator in $R$ software (version 3.6.2) was used to determine the grouping. Finally, the patients were randomly divided into primary $(n=2012)$ and validation $(n=859)$ cohorts based on the ratio of 7:3.

\section{Data extraction}

Data extraction was performed using PostgreSQL tools (V.1.13.1). The following information was extracted directly or calculated using data from the database: Age, gender, body mass index (BMI), Systemic inflammatory score (SIRS), laboratory variables, chronic medical conditions, comorbidities, length of stay in the intensive care unit, the time of AKI, administration of drugs. Laboratory variables including hemoglobin, platelet counts, glucose, serum creatinine, and albumin were measured during the first $24 \mathrm{~h}$ in the ICU. Chronic medical conditions included chronic obstructive pulmonary disease (COPD), chronic kidney disease (CKD), diabetes, coronary disease, malignant, chronic liver disease, and hypertension. Comorbidities included acute pancreatitis, lactic acidosis, heart failure, and hypotension. Notably, comorbidities and chronic medical conditions were collected based on the recorded ICD-9 codes in the MIMIC-III database. The drugs administrated to patients included vasoactive drugs, diuretic, aminoglycosides, lactated Ring, and human albumin. Variables associated with the risk of SA-AKI were assessed a priori based on scientific knowledge, clinical importance, and predictors identified in previously published articles $[9,14,25]$.

\section{Definitions and outcomes}

AKI during ICU stay was the primary outcome. AKI was defined following the Kidney Disease Improving Global Outcomes (KDGIO) criteria [26]. Considering that the 
patients might have used diuretics, AKI was defined only based on the change of serum creatinine value. Vasoactive drugs, diuretic, and aminoglycosides were defined as any vasoactive drugs, diuretic and aminoglycosides use during ICU stay for any reason. The severity of anemia was established following the reference standard of the World Health Organization (WHO) [27]. WHO international BMI cut-off points were used for BMI group categorization [28]: underweight $(\mathrm{BMI}<18.5)$, normal weight (BMI 18.5 to 24.9), overweight (BMI 25 to 29.9), and obesity $(\mathrm{BMI}>30)$.

\section{Statistical analysis}

Continuous variables were presented as interquartile ranges $\left(M\left(P_{25}, P_{75}\right)\right)$ unless indicated otherwise while categorical variables were presented as frequency and proportion of patients in each category. In the primary cohort, the assumption of linearity in the logistic for the continuous variable was assessed and univariate logistic analyses were used to analyze the relationships of relevant variables with SA-AKI. All variables with $\mathrm{P}<0.05$ in the univariate logistic analyses were further assessed by multivariable logistic regression using backward stepwise selection, where the variable with the largest p-value was eliminated at each step until all remaining variables had significant $p<0.05$. Multicollinearity was evaluated using variance inflation factors and there was no evidence of Multicollinearity. Nomograms predicting the risk of SA-AKI were determined using the independently selected significant variables. While ensuring the stability of prediction performance, a few features were removed to simplify the nomogram [23].

The performance of the nomograms was evaluated using the C-index [29] and calibration curves. The discriminative ability of the nomograms was evaluated by $\mathrm{C}$-index, where a C-index of 0.5 indicated the absence of discrimination, whereas a C-index of 1.0 suggested perfect discrimination. Calibration was assessed using calibration curves, graphic representation of the relationship between the frequency of observations and the probability of prediction, with a 1000-bootstrapped sample of the primary cohort. The final nomogram was verified in the validation cohort to assess the stability and generality of the nomogram. Moreover, the clinical utility of the final nomogram was evaluated using decision curve analysis by quantifying the net benefit at different threshold probabilities. The net benefit was calculated by subtracting the proportion of false positives from the proportion of true positives and weighing by the relative harm of foregoing treatment compared to the negative consequences of an unnecessary treatment [30].
For missing data, median imputation was used if the numbers were small $(<5 \%)$, while multiple imputations were used if the numbers with missing data were large.

Table 1 Characteristics of Patients in the Primary and Validation Cohorts after randomization

\begin{tabular}{|c|c|c|}
\hline Variable & Primary Cohort & Validation Cohort \\
\hline Age (years) & $69(56,81)$ & $68(55,81)$ \\
\hline Male, n (\%) & $1112(55.27)$ & $470(54.71)$ \\
\hline \multicolumn{3}{|l|}{ BMI, n (\%) } \\
\hline Normal & $624(31.01)$ & $254(29.57)$ \\
\hline Overweight & $396(19.68)$ & $203(23.63)$ \\
\hline Obesity & $923(45.87)$ & $366(42.61)$ \\
\hline Underweight & $69(3.43)$ & $36(4.19)$ \\
\hline LOS in ICU (days) & $3.7(1.9,8.9)$ & $2.9(1.9,9.9)$ \\
\hline SIRS & $3(3,4)$ & $3(3,4)$ \\
\hline \multicolumn{3}{|l|}{ Anemia, n (\%) } \\
\hline Normal & $491(24.40)$ & $176(20.49)$ \\
\hline Mild anemia & $1090(54.17)$ & $460(53.55)$ \\
\hline Moderate anemia & $428(21.27)$ & $222(25.84)$ \\
\hline Severe anemia & $3(0.15)$ & $1(0.12)$ \\
\hline Glucose ( mmol/L) & $7.0(4.3,11.9)$ & $7.0(4.2,11.4)$ \\
\hline Basline SCr ( umol/L) & $124(80,194)$ & $124(80,203)$ \\
\hline \multicolumn{3}{|l|}{ Albumin, n (\%) } \\
\hline$<30 \mathrm{~g} / \mathrm{L}$ & $932(46.32)$ & $420(48.89)$ \\
\hline$>=30 \mathrm{~g} / \mathrm{L}$ & $1080(53.68)$ & $439(51.11)$ \\
\hline \multicolumn{3}{|c|}{ Chronic medical conditions, n (\%) } \\
\hline COPD & $44(2.19)$ & $15(1.75)$ \\
\hline CKD & $338(16.8)$ & $165(19.21)$ \\
\hline Chronic liver disease & $105(5.22)$ & $59(6.87)$ \\
\hline Diabetes & $633(31.46)$ & $254(29.57)$ \\
\hline Coronary disease & $334(16.60)$ & $121(14.09)$ \\
\hline Malignant & $439(21.82)$ & $176(20.49)$ \\
\hline Hypertension & 798 ( 39.66 ) & $311(36.20)$ \\
\hline \multicolumn{3}{|l|}{ Comorbidity, n (\%) } \\
\hline Acute pancreatitis & $115(5.72)$ & $39(4.54)$ \\
\hline Lactic acidosis & $469(23.31)$ & $209(24.33)$ \\
\hline Heart failure & $631(31.36)$ & $253(29.45)$ \\
\hline Hypotension & $110(5.47)$ & $54(6.29)$ \\
\hline \multicolumn{3}{|l|}{ Medication, n (\%) } \\
\hline Vasoactive drugs & $891(44.28)$ & $388(45.17)$ \\
\hline Diuretic & $760(37.77)$ & $301(35.04)$ \\
\hline Aminoglycosides & $1516(75.35)$ & $645(75.09)$ \\
\hline Lactated Ring & $118(5.86)$ & $52(6.05)$ \\
\hline Human albumin & $222(11.03)$ & $91(10.59)$ \\
\hline
\end{tabular}

$B M I$ body mass index; SCr Serum creatinine; LOS in ICU length of stay in intensive care unit; SIRS Systemic inflammatory score; COPD chronic obstructive pulmonary disease; CKD chronic kidney disease 
All statistical analyses were performed using the $\mathrm{R}$ software (version 3.6.0). All tests were two-sided, with a significance level of $5 \%$.

\section{Results}

\section{Characteristics of patients with SA-AKI}

In total, 2,871 patients with sepsis were recruited and 1, 137 patients (39.6\%) positively tested for SA-AKI. The mean age of patients was 67 years, and a majority (55.1\%) were male. Patients were randomly assigned to primary (2,012 patients) or validation (859 patients) cohorts. Table 1 shows the characteristics of the patient in each cohort. The baseline clinical characteristics were similar between the two cohorts, with SA-AKI

Table 2 Univariate Logistic Regression Analysis of Factors relating to SAAKI in Primary Cohort

\begin{tabular}{|c|c|c|c|}
\hline Variable & OR & $95 \% \mathrm{Cl}$ & $P$-Value \\
\hline Age & 0.85 & $0.74-0.96$ & 0.119 \\
\hline Gender & 1.09 & $0.91-1.30$ & 0.371 \\
\hline $\mathrm{BMI}$ & 1.43 & $1.18-1.74$ & $<0.001$ \\
\hline LOS in ICU & 1.81 & $1.64-2.00$ & $<0.001$ \\
\hline SIRS & 1.34 & $1.19-1.50$ & $<0.001$ \\
\hline Basline SCr & 1.26 & $1.17-1.37$ & $<0.001$ \\
\hline Anemia & 3.38 & $2.27-5.04$ & $<0.001$ \\
\hline Glucose & 1.35 & $1.24-1.46$ & $<0.001$ \\
\hline Albumin & 1.74 & $1.45-2.08$ & $<0.001$ \\
\hline \multicolumn{4}{|c|}{ Chronic medical conditions } \\
\hline COPD & 1.11 & $0.61-2.03$ & 0.730 \\
\hline CKD & 1.96 & $1.55-2.47$ & $<0.001$ \\
\hline Diabetes & 1.44 & $1.19-1.75$ & $<0.001$ \\
\hline Chronic liver disease & 2.16 & $1.49-3.13$ & $<0.001$ \\
\hline Coronary disease & 1.46 & $1.15-1.86$ & 0.002 \\
\hline Malignant & 0.94 & $0.76-1.18$ & 0.610 \\
\hline Hypertension & 0.92 & $0.77-1.10$ & 0.365 \\
\hline \multicolumn{4}{|l|}{ Comorbidity } \\
\hline Acute pancreatitis & 1.49 & $1.00-2.21$ & 0.048 \\
\hline Lactic acidosis & 1.50 & $1.22-1.85$ & $<0.001$ \\
\hline Heart failure & 1.66 & $1.37-2.01$ & $<0.001$ \\
\hline Hypotension & 1.05 & $0.71-1.55$ & 0.799 \\
\hline \multicolumn{4}{|l|}{ Medication } \\
\hline Vasoactive drugs & 2.27 & $1.89-2.72$ & $<0.001$ \\
\hline Diuretic & 0.88 & $0.74-1.06$ & 0.191 \\
\hline Aminoglycosides & 1.56 & $1.26-1.93$ & $<0.001$ \\
\hline Lactated Ring & 0.90 & $0.62-1.30$ & 0.569 \\
\hline Human albumin & 2.78 & $2.08-3.71$ & $<0.001$ \\
\hline
\end{tabular}

$B M I$ body mass index; $S C r$ Serum creatinine; LOS in ICU length of stay in intensive care unit; SIRS Systemic inflammatory score; COPD chronic obstructive pulmonary disease ;CKD chronic kidney disease proportions of 39.2 and $40.6 \%$ in the primary and validation cohorts, respectively.

\section{Model specifications and predictors of SA-AKI}

Established risk factors, AKI, and demographic characteristics of clinical importance were selected as candidate variables for the prediction model. Variables relevant to SA-AKI in the primary cohort were subjected to univariate and multivariate logistic regression analyses, and the result is illustrated in Tables 2 and 3. Final statistical analysis identified 12 predictors for SA-AKI, including $\mathrm{BMI}$, LOS in ICU, baseline $\mathrm{SCr}$, glucose, anemia incidence, $C K D$, heart failure, coronary disease, chronic liver disease, and the use of human albumin or vasoactive drugs.

Nomograms and model performance in the primary cohort Nomograms for SA-AKI incorporating significant predictive factors from the multivariate analysis were established (Figs. 1 and 2). Nomogram 1 included 12 significant predictors for SA-AKI prediction (Fig. 1), depicting moderate discrimination in prediction with an unadjusted C-Index of 0.773 (95 \% Cl, 0.752-0.794).

Table 3 Results of the forward stepwise logistic regression analysis of SA-AKI in Primary Cohort

\begin{tabular}{|c|c|c|c|}
\hline Variable & OR & $95 \% \mathrm{Cl}$ & $P$-Value \\
\hline BMI & 1.40 & $1.13-1.75$ & 0.003 \\
\hline LOS in ICU & 1.65 & $1.50-1.82$ & $<0.001$ \\
\hline SIRS & 1.11 & $0.98-1.26$ & 0.114 \\
\hline Basline SCr & 1.19 & $1.08-1.30$ & $<0.001$ \\
\hline Anemia & 2.24 & $1.42-3.55$ & $<0.001$ \\
\hline Glucose & 1.21 & $1.11-1.33$ & $<0.001$ \\
\hline Albumin & 1.33 & $1.08-1.63$ & 0.007 \\
\hline \multicolumn{4}{|c|}{ Chronic medical conditions } \\
\hline CKD & 1.74 & $1.31-2.30$ & $<0.001$ \\
\hline Chronic liver disease & 1.75 & $1.13-2.71$ & 0.012 \\
\hline Diabetes & 1.04 & $0.83-1.31$ & 0.752 \\
\hline Coronary disease & 1.44 & $1.09-1.91$ & 0.012 \\
\hline \multicolumn{4}{|l|}{ Comorbidity } \\
\hline Acute pancreatitis & 0.88 & $0.56-1.39$ & 0.590 \\
\hline Lactic acidosis & 1.06 & $0.84-1.35$ & 0.613 \\
\hline Heart failure & 1.29 & $1.03-1.62$ & 0.025 \\
\hline \multicolumn{4}{|l|}{ Medication } \\
\hline Vasoactive drugs & 2.15 & $1.74-2.66$ & $<0.001$ \\
\hline Aminoglycosides & 1.15 & $0.90-1.48$ & 0.267 \\
\hline Human albumin & 2.55 & $1.83-3.56$ & $<0.001$ \\
\hline
\end{tabular}

$B M I$ body mass index; SCr Serum creatinine; LOS in ICU length of stay in intensive care unit; SIRS Systemic inflammatory score; COPD chronic obstructive pulmonary disease; $C K D$ chronic kidney disease 




However, nomogram 1, combining 12 predictors was cumbersome. Besides, BMI, albumin, CKD, heart failure, coronary disease, chronic liver disease, and human albumin predictors demonstrated insignificant influence on the point in nomogram 1. For effective visualization, a few predictors were reduced to simplify the nomogram. Nomogram 2 (Fig. 2) including factors with LOS in ICU, baseline $\mathrm{SCr}$, glucose, anemia, and vasoactive drugs maintained similar discrimination (C-index 0.752, $95 \% \mathrm{Cl}$ [0.730-0.774]).

Therefore, nomogram 2 was identified as the effective predictive model. To use nomogram 2 , a vertical line should be delineated to the point raw to assign point values for each predictor. Each patient obtains a total point by plus the points of five predictors in nomogram 2. For instance, a patient with mild anemia, the glucose of $10 \mathrm{mmol} / \mathrm{L}$, is using vasoactive drugs, baseline SCr of $200 \mathrm{umol} / \mathrm{L}$ and 10 days Los in ICU, total point taken from nomogram 2 of the patient is $38(5+4+12+6+11=38)$, and the risk of SA-AKI probability is $60 \%$. The weights for each feature are list in Table 4 for calculation without a nomogram. The bootstrap-corrected C-index for the prediction nomogram 2 was 0.749 and the calibration 


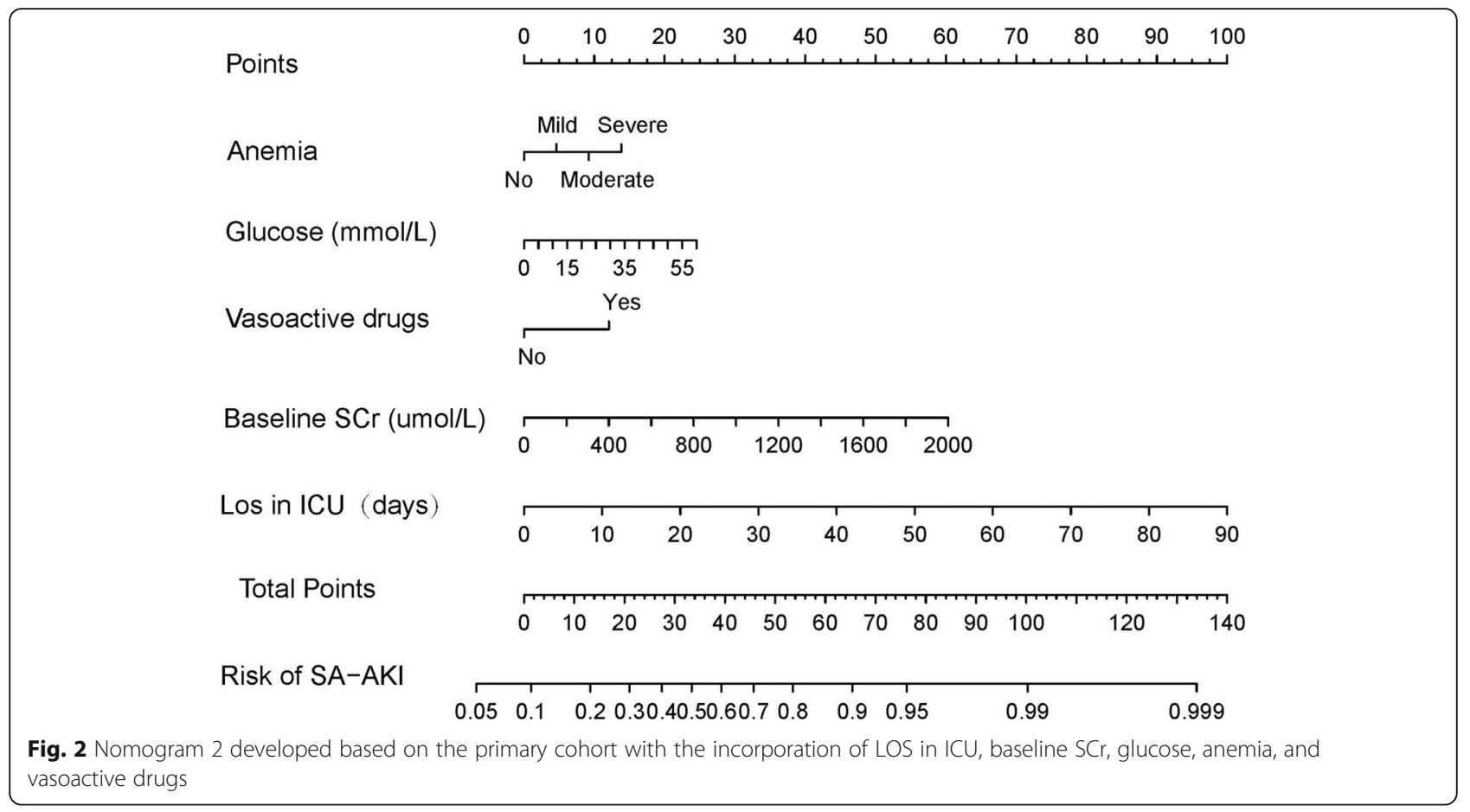

plot for the probability of SA-AKI revealed a good correlation between nomogram 2 prediction and actual observation (Fig. 3), indicating moderate discrimination by our final model.

\section{External validation of the nomogram 2 in the validation cohort}

In the validation cohort, nomogram 2 displayed a Cindex of 0.757 (95\% CI 0.724-0.790) for estimation of SA-AKI risk. Also, there was a good calibration curve for risk estimation (Fig. 4).

\section{Clinical use of nomogram 2}

The decision curve analysis (DCA) for nomogram 2 and the individual predictor is illustrated in Fig. 5. The DCA revealed that nomogram 2 would effectively predict SAAKI if the threshold probability of SA-AKI is between 15 and $80 \%$. Within this range, the predictive effect of the nomogram is better than that of a single predictor, respectively.

\section{Discussion}

Sepsis Associated Acute Kidney Injury (SA-AKI) is a frequent complication among critically ill patients causing alarming mortality and morbidity. SA-AKI has a higher risk of in-hospital death and longer hospital stay than AKI caused by other factors [31]. However, crude markers of renal function including $\mathrm{SCr}$ and Urine output are not effective in early diagnosis. This study developed and verified a diagnostic nomogram for predicting SA-AKI in critically ill patients. The nomogram incorporates five items, including LOS in ICU, baseline $\mathrm{SCr}$, glucose, anemia, and vasoactive drugs which can be used to diagnose critically ill patients, enabling individualized decisions on the surveillance and treatment. Our findings are valuable since the nomogram was established based on a large database of critically ill patients. Additionally, in contrast with previously proposed models based on the clinical risk factors [25], the current nomogram has stable performance with ease of visualization.

Inflammation, oxidative stress, microvascular endothelial dysfunction, and renal tubular epithelial cell injury are the potential mechanisms of SA-AKI, however, its pathogenesis remains elusive [9]. The occurrence and development of these mechanisms [4, $9,20]$ are potentially attributed to the variables in our model which might promote or balance each other and influence the process of SA-AKI. Therefore, predicting the occurrence of SA-AKI via these clinical variables is of importance.

Our study considered a wide array of clinical risk predictors previously linked to SA-AKI [4, 9, 14]. Specifically, in the medical treatment, we noted no relationship between diuretic, aminoglycosides, and SA-AKI. In contrast, data on SA-AKI demonstrated that diuretics might not be conducive to the prevention of AKI [4], and 
Table 4 the points for predictors

Predictor
Anemia
Normal
Mild anemia
Moderate anemia
Severe anemia
Glucose ( $\mathrm{mmol} / \mathrm{L})$
5
10
15
20
25
30
35
40
45
50
55
60

Vasoactive drugs

No

Yes

baseline SCr (umol/L)

200

400

600

800

1000

1200

1400

1600

1800

2000

LOS in ICU (days)

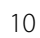

20

30

40

50

60

70

80

90

Total points

1
Table 4 the points for predictors (Continued)

\begin{tabular}{ll}
\hline Predictor & points \\
\hline 13 & $20.0 \%$ \\
21 & $30.0 \%$ \\
27 & $40.0 \%$ \\
33 & $50.0 \%$ \\
39 & $60.0 \%$ \\
46 & $70.0 \%$ \\
54 & $80.0 \%$ \\
65 & $90.0 \%$ \\
76 & $95.0 \%$ \\
100 & $99.0 \%$ \\
\hline 34 & $99.9 \%$ \\
\hline
\end{tabular}

aminoglycosides are harmful to renal function. Predictors associated with AKI extensively vary, but rarely for sepsis and limited studies have integrated them with clinical prediction models. Recently, Zhou et al. analyzed 2,617 patients with sepsis and constructed a comprehensive risk score comprising 16 factors to predict SA-AKI [25], and lacked analysis of drug use (For example, vasoactive drugs), which might be unspecific for clinical use. The multivariable model including all contrast-enhanced ultrasonography variables created by Liu et al. was also complex and unsuitable for quick risk assessment [32]. This work differs from the previous simple multi-factor analysis [33] in that we removed predictors with little effect on the points in the nomogram to simplify the model for clinical application. Furthermore, none of the existing risk models was estimated by the DCA for their clinical utility. To our knowledge, this is the first risk model to consider previously proposed risk factors to develop a nomogram for prediction the prognosis of SA-AKI.

Since the treatment strategies are potentially heterogeneous, accurate AKI risk stratification of the critically ill patients with sepsis is critical. Although the prevention of AKI in critically ill patients has formed a certain consensus, instances, where a recommendation cannot or should not be followed for an individual patient, have been reported [11]. Rather than using biomarkers or clinical risk factors alone, derived based on large population or cohort data, the nomogram provides a more individualized admonition for risk information to septic patients. For example, the European Society of Intensive Care Medicine suggests a mandatory review of all medications with cessation of nephrotoxic ones in addition to the treatment measures [11]. However, many aspects may affect the treatment strategy of septic patients and 


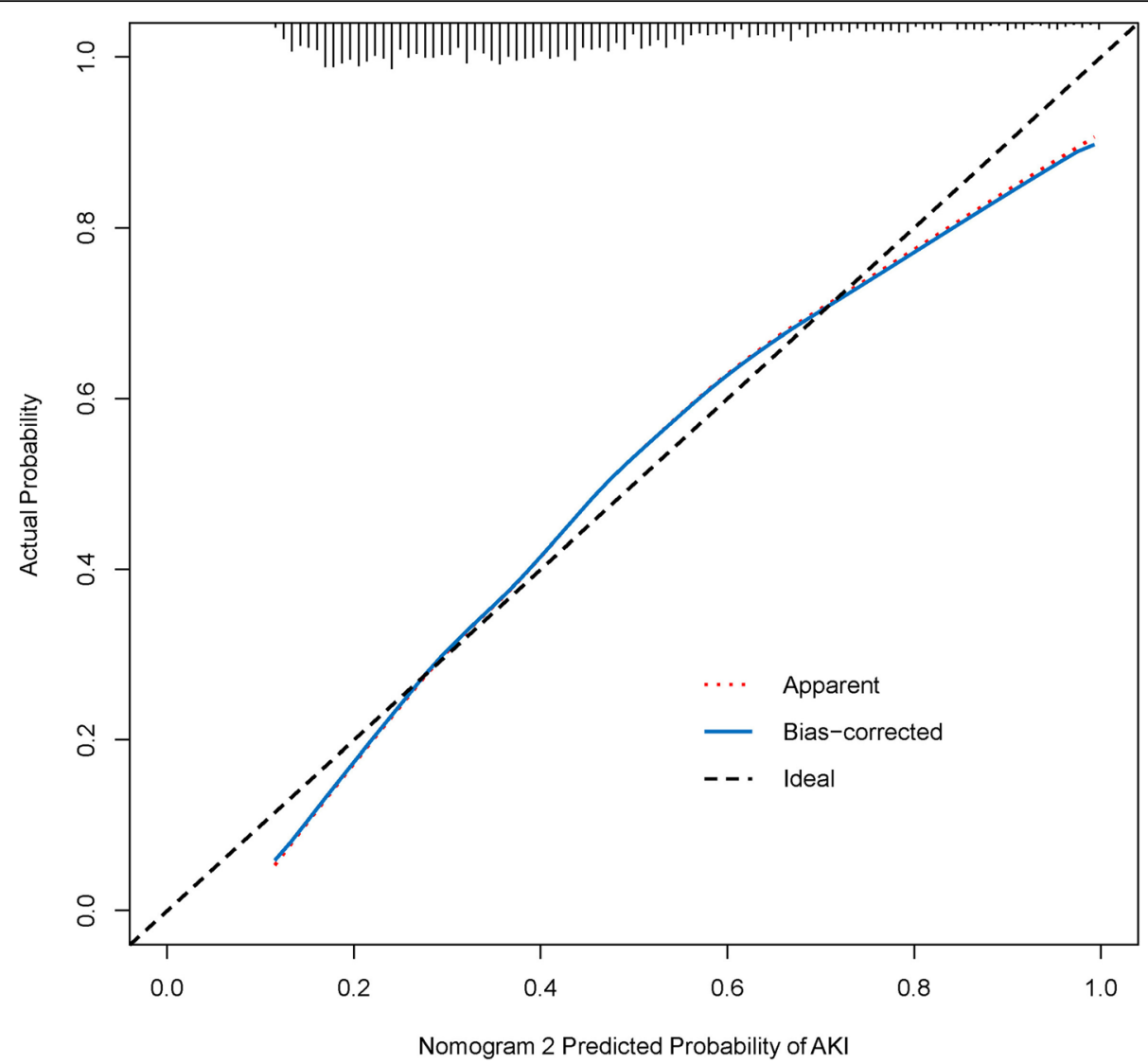

Fig. 3 Calibration curves for nomogram 2 in the primary cohort. The blue dotted line represents the entire cohort $(n=2,012)$, and the red solid line depicts the result after bias-correction by bootstrapping (1000 repetitions), indicating the performance of nomogram 2

the lesions changed quickly, clinicians depend on their clinical experience. Clinicians may be more accurate in selecting treatment strategies for a higher probability of benefiting from treatments, using a nomogram with clinical factors.

The most important and final argument for the use of the nomogram is based on individual needs for additional investigation or care [22]. Although is have good prediction performance, discrimination and calibration, the nomogram cannot capture the clinical consequences of miscalibration or a particular level of discrimination. The DCA is a valuable decision-making tool when different means are compared with their clinical value [34-36]. Therefore, to justify its clinical utility, DCA was applied to unravel the usefulness of nomogram 2 decisions. This novel method provides insights into clinical consequences based on threshold probabilities, where a net benefit is derived [34]. The decision curve revealed that nomogram 2 caused a positive net benefit with a threshold probability of between 15 and $80 \%$. For example, if the threshold probability of a patient is $40 \%$, the net benefit would be $15 \%$ when nomogram 2 is used to predict AKI, hence more benefit than either the treatnone or the treat-all scheme.

Despite these promising findings, this paper has some limitations. First, the nomogram was retrospectively constructed and new biomarkers were not included in the analysis, potentially reducing the performance of the model. Secondly, the urine standard was not used in the diagnosis of AKI since urine volume data may be unreliable due to the use of diuretics. This may reduce the overall incidence rate of AKI. Thirdly, the missing data were settled with median imputation and multiple imputation techniques, potentially decreasing the accuracy and C-index of the final model. Nonetheless, data for these predictors can easily be obtained in the intensive care units and not an obstacle in implementing the nomogram. Furthermore, these disadvantages are natural in any retrospective studies, and population-based research thereby increasing concerns on the stability of the results. Nevertheless, this nomogram may somewhat help clinicians make reasonable risk judgments and treatment strategies in the absence of high-quality SA-AKI prediction tools. 




Fig. 4 Calibration curves for nomogram 2 in the validation cohort. The blue dotted line represents the entire cohort $(n=859)$, and the red solid line is the result after bias correction by bootstrapping (1000 repetitions), indicating the performance of nomogram 2

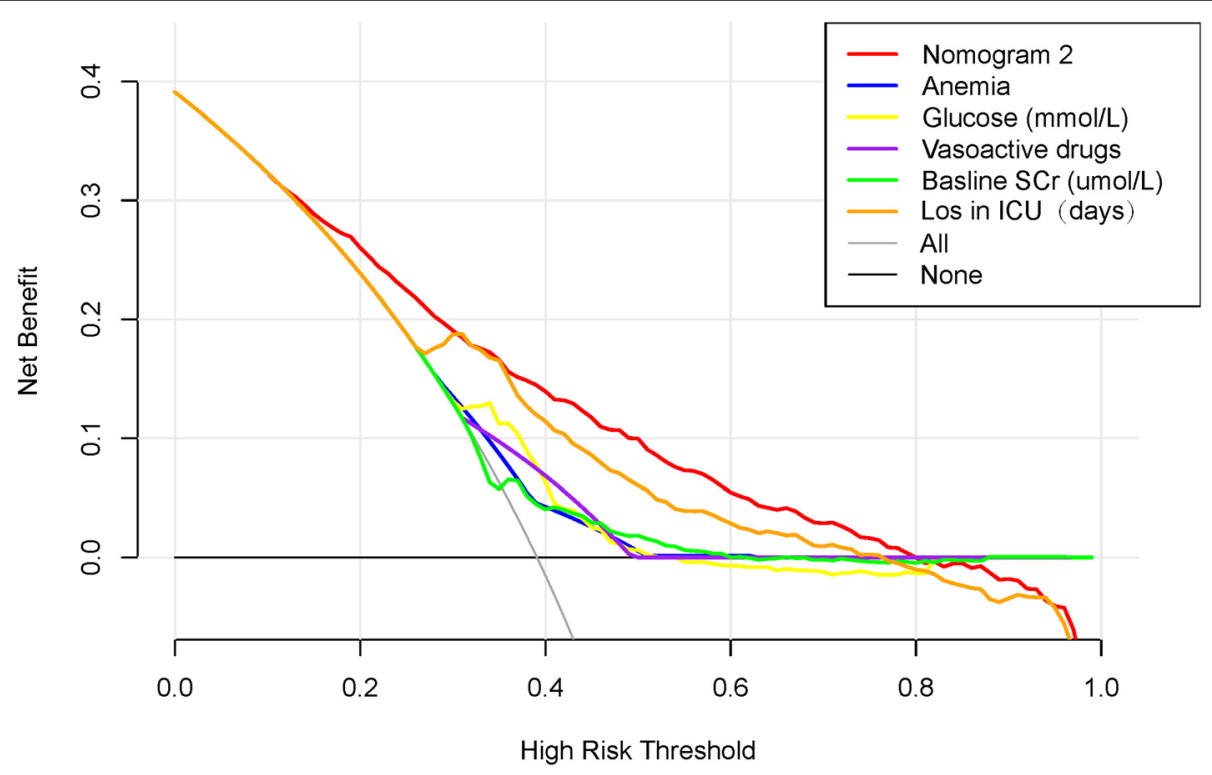

Fig. 5 Decision curve analysis for nomogram 2, LOS in ICU, baseline SCr, glucose, anemia, and vasoactive drugs 


\section{Conclusions}

This study developed and verified an AKI risk prediction nomogram applied to critically ill patients with sepsis, which may partially help clinicians make reasonable risk decisions and treatment strategies. Nonetheless, further verification using external data is essential to enhance the applicability of this nomogram in clinical practice.

\section{Abbreviations}

AKI: Acute kidney injury; SA-AKI: Sepsis associated-AKI; MIMIC-III: Medical Information Mart for Intensive Care III; BMI: Body mass index; SCr: Serum creatinine; LOS in ICU: Length of stay in intensive care unit; SIRS: Systemic inflammatory score; COPD: Chronic obstructive pulmonary disease; CKD: Chronic kidney disease

\section{Acknowledgements}

Not application.

\section{Authors' contributions}

SY and TS conceived the study. SY, TS and LH carried out the research. SY, LHF and TL analysed the data. SY, L-HF and TL wrote the paper. All authors read and approved the final manuscript.

\section{Funding}

This work was supported by a grant from scientific research and technology development plan of Baise City (No. baike 20204754), Self-financing project of Guangxi Zhuang Autonomous Region Health Committee (NO: Z20191091) and self-financing project of the Traditional Chinese Medicine Administration of Guangxi Zhuang Autonomous Region (NO. GZZC2020234).

\section{Availability of data and materials}

The datasets used and/or analysed during the current study are available from the corresponding author upon reasonable request.

\section{Declarations}

\section{Ethics approval and consent to participate}

The establishment of MIMIC-III (v1.4) was approved by the institutional review boards of the Beth Israel Deaconess Medical Center (Boston, MA) and Massachusetts Institute of Technology (Cambridge, MA), thus, this study was granted a waiver of informed consent.

\section{Consent for publication}

Not Applicable.

\section{Competing interests}

None of the authors have any conflicts of interests to declare.

\section{Author details}

'Department of Academic Affairs Office, YouJiang Medical University for Nationalities, Baise, China. '2Department of ECG Diagnostics, The People's Hospital of Guangxi Zhuang Autonomous Region, Nanning, China. ${ }^{3}$ Department of Comprehensive Internal Medicine, The Affiliated Tumor Hospital of Guangxi Medical University, Nanning, China. ${ }^{4}$ Department of President's Office, YouJiang Medical University for Nationalities, Baise, China.

Received: 1 February 2021 Accepted: 28 April 2021

Published online: 10 May 2021

\section{References}

1. Rhodes A, Evans LE, Alhazzani W, Levy MM, Antonelli M, Ferrer R, Kumar A, Sevransky JE, Sprung CL, Nunnally ME et al: Surviving Sepsis Campaign: International Guidelines for Management of Sepsis and Septic Shock: 2016 Intensive care medicine 2017, 43(3):304-377.

2. Kadri SS, Rhee C, Strich JR, Morales MK, Hohmann S, Menchaca J, Suffredin AF, Danner RL, Klompas M: Estimating Ten-Year Trends in Septic Shock Incidence and Mortality in United States Academic Medical Centers Using Clinical Data. Chest 2017, 151(2):278-285.
3. Wang HE, Jones AR, Donnelly JP: Revised National Estimates of Emergency Department Visits for Sepsis in the United States. Critical care medicine 2017, 45(9):1443-1449.

4. Poston JT, Koyner JL: Sepsis associated acute kidney injury. BMJ (Clinical research ed) 2019, 364:k4891.

5. Skube SJ, Katz SA, Chipman JG, Tignanelli CJ: Acute Kidney Injury and Sepsis. Surgical infections 2018, 19(2):216-224.

6. Hoste EA, Bagshaw SM, Bellomo R, Cely CM, Colman R, Cruz DN, Edipidis K, Forni LG, Gomersall CD, Govil D et al: Epidemiology of acute kidney injury in critically ill patients: the multinational AKI-EPI study. Intensive care medicine 2015, 41(8):1411-1423.

7. Gómez H, Kellum JA: Sepsis-induced acute kidney injury. Current opinion in critical care 2016, 22(6):546-553.

8. Bouchard J, Acharya A, Cerda J, Maccariello ER, Madarasu RC, Tolwani AJ, Liang X, Fu P, Liu ZH, Mehta RL: A Prospective International Multicenter Study of AKI in the Intensive Care Unit. Clinical journal of the American Society of Nephrology: CJASN 2015, 10(8):1324-1331.

9. Bellomo R, Kellum JA, Ronco C, Wald R, Martensson J, Maiden M, Bagshaw SM, Glassford NJ, Lankadeva Y, Vaara ST et al: Acute kidney injury in sepsis. Intensive care medicine 2017, 43(6):816-828.

10. Doyle JF, Forni LG: Update on sepsis-associated acute kidney injury: emerging targeted therapies. Biologics: targets \& therapy 2016, 10:149-156.

11. Joannidis M, Druml W, Forni LG, Groeneveld ABJ, Honore PM, Hoste E, Ostermann M, Oudemans-van Straaten HM, Schetz M: Prevention of acute kidney injury and protection of renal function in the intensive care unit: update 2017: Expert opinion of the Working Group on Prevention, AKI section, European Society of Intensive Care Medicine. Intensive care medicine 2017, 43(6):730-749

12. Khwaja A: KDIGO Clinical Practice Guidelines for Acute Kidney Injury. Nephron 2012, 120(4):c179-c184.

13. Topol EJ: Deep learning detects impending organ injury in the clinic. Nature 2019, 572(7767):36-37

14. Liu J, Xie H, Ye Z, Li F, Wang L: Rates, predictors, and mortality of sepsisassociated acute kidney injury: a systematic review and meta-analysis. BMC nephrology 2020, 21(1):318

15. Prowle JR: Measurement of AKI biomarkers in the ICU: still striving for appropriate clinical indications. Intensive care medicine 2015, 41(3):541-543.

16. Hoste EAJ, Kellum JA, Selby NM: Global epidemiology and outcomes of acute kidney injury. Nature reviews Nephrology 2018, 14(10):607-625.

17. Mercado MG, Smith DK, Guard EL: Acute Kidney Injury: Diagnosis and Management. American family physician 2019, 100(11):687-694.

18. Göcze I, Jauch D, Götz M, Kennedy P, Jung B, Zeman F, Gnewuch C, Graf BM, Gnann W, Banas B et al: Biomarker-guided Intervention to Prevent Acute Kidney Injury After Major Surgery: The Prospective Randomized BigpAK Study. Annals of surgery 2018, 267(6):1013-1020.

19. Pickkers P, Mehta RL, Murray PT, Joannidis M, Molitoris BA, Kellum JA, Bachler M, Hoste EAJ, Hoiting O, Krell K et al: Effect of Human Recombinant Alkaline Phosphatase on 7-Day Creatinine Clearance in Patients With SepsisAssociated Acute Kidney Injury: A Randomized Clinical Trial. Jama 2018, 320(19):1998-2009.

20. Koyner JL, Carey KA, Edelson DP, Churpek MM: The Development of a Machine Learning Inpatient Acute Kidney Injury Prediction Model. Critical care medicine 2018, 46(7):1070-1077.

21. Tomašev N, Glorot X, Rae JW, Zielinski M, Askham H, Saraiva A, Mottram A, Meyer C, Ravuri S, Protsyuk I et al: A clinically applicable approach to continuous prediction of future acute kidney injury. Nature 2019, 572(7767): 116-119.

22. Feng LH, Bu KP, Ren S, Yang Z, Li BX, Deng CE: Nomogram for Predicting Risk of Digestive Carcinoma Among Patients with Type 2 Diabetes. Diabetes, metabolic syndrome and obesity: targets and therapy 2020, 13:1763-1770.

23. lasonos A, Schrag D, Raj GV, Panageas KS: How to build and interpret a nomogram for cancer prognosis. Journal of clinical oncology: official journal of the American Society of Clinical Oncology 2008, 26(8):1364-1370.

24. Johnson AEW, Pollard TJ, Shen L, Lehman LWH, Feng M, Ghassemi M, Moody B, Szolovits P, Anthony Celi L, Mark RG: MIMIC-III, a freely accessible critical care database. Scientific Data.

25. Zhou J, Bai Y, Wang X, Yang J, Fu P, Cai D, Yang L: A simple risk score for prediction of sepsis associated-acute kidney injury in critically ill patients. Journal of nephrology 2019, 32(6):947-956.

26. Kellum JA, Lameire N: Diagnosis, evaluation, and management of acute kidney injury: a KDIGO summary (Part 1). Critical Care 2013, 17(1):204. 
27. WHO: Haemoglobin concentrations for the diagnosis of anaemia and assessment of severity Vitamin and Mineral Nutrition Information System. Geneva, Switzerland 2011.

28. Organization WH: Obesity and overweight fact sheet. Geneva, Switzerland: World Health Organization. 2018. In.; 2020.

29. Harrell FE, Califf RM, Pryor DB, Lee KL, Rosati RA: Evaluating the yield of medical tests. Jama 1982, 247(18):2543-2546.

30. Vickers AJ, Cronin AM, Elkin EB, Gonen M: Extensions to decision curve analysis, a novel method for evaluating diagnostic tests, prediction models and molecular markers. BMC Med Inform Decis Mak 2008, 8(1):53.

31. Zhi DY, Lin J, Zhuang HZ, Dong L, Ji XJ, Guo DC, Yang XW, Liu S, Yue Z, Yu SJ et al: Acute Kidney Injury in Critically III Patients with Sepsis: Clinical Characteristics and Outcomes. Journal of investigative surgery: the official journal of the Academy of Surgical Research 2019, 32(8):689-696.

32. Liu N, Zhang Z: Protocol for a prospective observational study on the association of variables obtained by contrast-enhanced ultrasonography and sepsis-associated acute kidney injury. 2019, 9(7):e023981.

33. Fan $C$, Ding $X$, Song Y: A new prediction model for acute kidney injury in patients with sepsis. Annals of palliative medicine 2020.

34. Collins GS, Reitsma JB, Altman DG, Moons KG: Transparent reporting of a multivariable prediction model for individual prognosis or diagnosis (TRIPOD): the TRIPOD statement. Br J Surg 2015, 102(3):148-158.

35. Van Calster B, Vickers AJ: Calibration of risk prediction models: impact on decision-analytic performance. Med Decis Making 2015, 35(2):162-169.

36. Localio AR, Goodman S: Beyond the usual prediction accuracy metrics: reporting results for clinical decision making. Ann Intern Med 2012, 157(4): 294-295.

\section{Publisher's Note}

Springer Nature remains neutral with regard to jurisdictional claims in published maps and institutional affiliations.

Ready to submit your research? Choose BMC and benefit from:

- fast, convenient online submission

- thorough peer review by experienced researchers in your field

- rapid publication on acceptance

- support for research data, including large and complex data types

- gold Open Access which fosters wider collaboration and increased citations

- maximum visibility for your research: over $100 \mathrm{M}$ website views per year

At $\mathrm{BMC}$, research is always in progress.

Learn more biomedcentral.com/submissions 